\title{
Common fixed points for some generalized contraction pairs in convex metric spaces
}

Mohammad Moosaei*

\section{"Correspondence:}

m.moosaei@basu.ac.ir

Department of Mathematics, Bu-Ali

Sina University, Hamedan, Iran

\begin{abstract}
The present study focuses on proving the existence of coincidence points for self-mappings satisfying a generalized contractive condition within the framework of convex metric spaces. The existence of common fixed points for weakly compatible self-mappings as well as Banach operator pairs under certain generalized contractions in a convex metric space is also established.
\end{abstract}

MSC: $47 \mathrm{H} 09 ; 47 \mathrm{H} 10 ; 47 \mathrm{H} 19 ; 54 \mathrm{H} 25$

Keywords: Banach operator pairs; coincidence points; common fixed points; compatible mappings; convex metric spaces; fixed points; weakly compatible pair

\section{Introduction and preliminaries}

In 1970, Takahashi [1] introduced the notion of convexity in metric spaces and proved that all normed spaces and their convex subsets are convex metric spaces. He also gave some examples of the convex metric spaces which are not embedded in any normed/Banach spaces. Afterward Guay, Singh and Whittield [2], Beg and Azam [3], Beg, Azam, Ali and Minhas [4], Shimizu and Takahashi [5], Ciric [6], Beg [7, 8], Beg and Abbas [9], and many other authors have studied fixed point theorems in convex metric spaces.

In this paper, we introduce $(\alpha, \beta, \gamma, \eta)$-generalized contraction pairs and study the existence of a coincidence point for such pairs in a convex metric space under certain conditions (see Theorem 2.2). Consequently, we prove the existence of a common fixed point for weakly compatible mappings and also Banach operator pairs which are $(\alpha, \beta, \gamma, \eta)$ generalized contraction pairs (see Theorem 2.3 and Theorem 2.5).

We now review notations and definitions needed. We denote by $\mathbb{N}$ and $\mathbb{R}$ the set of natural numbers and the set of real numbers, respectively. We also denote by $I$ the identity mapping. In what follows, $(X, d)$ is a metric space, and $C$ is a nonempty subset of $X$.

Definition 1.1 Let $S$ and $T$ be two self-mappings of $C$. A point $x$ of $C$ is called

(i) a fixed point of $T$ if $T x=x$,

(ii) a common fixed point of the pair $(S, T)$ if $S x=T x=x$, and

(iii) a coincidence point of the pair $(S, T)$ if $S x=T x$.

The set of fixed points of $T$ is denoted by $F(T)$. The set of common fixed points (respectively, coincidence points) of the pair $(S, T)$ is denoted by $F(S, T)$ (respectively, $C(S, T)$ ). Note that $C(I, T)=F(T)$.

Definition 1.2 Let $S$ and $T$ be two self-mappings of $C$. The mapping $T$ is called

\section{照 Springer}

C2014 Moosaei; licensee Springer. This is an Open Access article distributed under the terms of the Creative Commons Attribution License (http://creativecommons.org/licenses/by/2.0), which permits unrestricted use, distribution, and reproduction in any medium, provided the original work is properly cited. 
(i) a contraction if there exists $k \in[0,1)$ such that $d(T x, T y) \leq k d(x, y)$ for all $x, y \in C$,

(ii) an $S$-contraction if there exists $k \in[0,1)$ such that $d(T x, T y) \leq k d(S x, S y)$ for all $x, y \in C$,

(iii) nonexpansive if $d(T x, T y) \leq d(x, y)$ for all $x, y \in C$, and

(iv) $S$-nonexpansive if $d(T x, T y) \leq d(S x, S y)$ for all $x, y \in C$.

Definition 1.3 Let $S$ and $T$ be two self-mappings of $C$. The pair $(S, T)$ is said to be

(i) commuting if $S T x=T S x$ for all $x \in C$,

(ii) $R$-weakly commuting [10] if there exists $R>0$ such that $d(S T x, T S x) \leq R d(S x, T x)$ for all $x \in C$. If $R=1$, then the mappings are called weakly commuting [11],

(iii) compatible [12] if $\lim _{n \rightarrow \infty} d\left(S T x_{n}, T S x_{n}\right)=0$, whenever $\left\{x_{n}\right\}_{n=1}^{\infty}$ is a sequence in $C$ such that $\lim _{n \rightarrow \infty} S x_{n}=\lim _{n \rightarrow \infty} T x_{n}=x$ for some $x \in C$, and

(iv) weakly compatible if they commute on $C(S, T)$ i.e. $S T x=T S x$ for all $x \in C(S, T)$ (see $[13,14]$ for more details).

It is well known that commuting mappings are weakly commuting, and weakly commuting mappings are $R$-weakly mappings. Moreover, $R$-weakly mappings are compatible, and compatible mappings are weakly compatible.

The following example shows that the converses of the above results are not true in general.

Example 1.4 Let $X=\mathbb{R}$ with the usual metric $d(x, y)=|x-y|$ for all $x, y \in X$, we have:

(1) Let $C=[0,1]$. Let $S x=x^{2}$ and $T x=\frac{x^{2}}{2}$ for all $x \in C$. It is trivial that $S$ and $T$ are weakly commuting but are not commuting.

(2) Let $C=[0, \infty]$. Consider $S x=2 x-1$ and $T x=x^{2}$ for all $x \in C$. Then $S$ and $T$ are 2 -weakly commuting but are not weakly commuting (see [10]).

(3) Let $C=X, S x=x^{3}, T x=2 x^{3}, x \in C$. Then $S$ and $T$ are compatible but are not $R$-weakly commuting (see $[12,15,16]$ for more details).

(4) Let $C=[0,10]$, and define self-mappings $S$ and $T$ of $C$ by $S(1)=1, S(x)=4$ if $1<x<6, S(x)=1$ if $6 \leq x \leq 10$, and $T(1)=1, T(x)=3$ if $1<x<6, T(x)=x-5$ if $6 \leq x \leq 10$. For sequence $\left\{x_{n}\right\}_{n=1}^{\infty}$ defined by $x_{n}=6+\frac{1}{n}, n \geq 1$, we have $\lim _{n \rightarrow \infty} S x_{n}=\lim _{n \rightarrow \infty} T x_{n}=1$, but $\lim _{n \rightarrow \infty} d\left(S T x_{n}, T S x_{n}\right)=3 \neq 0$. So the mappings $S$ and $T$ are not compatible. It is easy to see that $S$ and $T$ are weakly compatible.

Definition 1.5 Let $C$ be a nonempty subset of a metric space $(X, d)$, and let $S$ and $T$ be self-mappings of $C$. The ordered pair $(S, T)$ is called a Banach operator pair if the set $F(T)$ is $S$-invariant, namely $S(F(T)) \subseteq F(T)$ (see [17]).

It is easy to see that if the mappings $S$ and $T$ are commuting, then the pair $(S, T)$ is a Banach operator pair, but the converse is not true in general (see Example 1(ii) of [17]). If $(S, T)$ is a Banach operator pair, then $(T, S)$ need not be a Banach operator pair (see $[17,18])$.

Definition 1.6 Let $S$ and $T$ be two self-mappings of a nonempty subset $C$ of a metric space $(X, d)$. Consider $(\alpha, \beta, \gamma, \eta) \in \mathbb{R}^{4}$ with

$$
2 \beta+\gamma-|\gamma|-\alpha \leq \eta<\alpha+4 \beta+3 \gamma-|\gamma|, \quad \beta+\gamma \leq 0 .
$$


We call the ordered pair $(S, T)$ is an $(\alpha, \beta, \gamma, \eta)$-generalized contraction pair if the following inequality holds:

$$
\alpha d(T x, T y)+\beta(d(S x, T x)+d(S y, T y))+\gamma(d(S x, T y)+d(S y, T x)) \leq \eta d(S x, S y)
$$

for all $x, y \in C$. The mapping $T$ is called an $(\alpha, \beta, \gamma, \eta)$-generalized contraction if $(I, T)$ is an $(\alpha, \beta, \gamma, \eta)$-generalized contraction pair.

If $(S, T)$ is an $(\alpha, 0,0, \eta)$-generalized contraction pair, then $T$ is an $S$-contraction. In particular, if $S=I$, then $T$ is a contraction. If $(S, T)$ is an $(\alpha, \beta, \gamma, \eta)$-generalized contraction pair, then $(T, S)$ need not be an $(\alpha, \beta, \gamma, \eta)$-generalized contraction pair in general (see Example 2.4).

Definition 1.7 Let $(X, d)$ be a metric space. A mapping $W: X \times X \times[0,1] \rightarrow X$ is said to be a convex structure on $X$ if

$$
d(u, W(x, y, \lambda)) \leq \lambda d(u, x)+(1-\lambda) d(u, y)
$$

for each $x, y, u \in X$ and $\lambda \in[0,1]$ (see [1]). A metric space $(X, d)$ together with a convex structure $W$ is called a convex metric space. A nonempty subset $C$ of $X$ is said to be convex if $W(x, y, \lambda) \in C$ for all $x, y \in C$ and $\lambda \in[0,1]$ (see $[1,19]$ ).

Let $X$ be a convex metric space. The open balls and the closed balls are convex subsets of $X$. If $\left\{C_{\alpha}\right\}_{\alpha \in J}$ is family of convex subsets of $X$, then $\bigcap_{\alpha \in J} C_{\alpha}$ is a convex subset of $X$ (see $[1,19]$ for more details). All normed spaces and their convex subsets are convex metric spaces. But there are some examples of convex metric spaces which are not embedded in any normed space (see [1]).

Definition 1.8 Let $C$ be a convex subset of a convex metric space $X$ with the structure $W$. A self-mapping $T$ of $C$ is said to be affine if $T(W(x, y, \lambda))=W(T x, T y, \lambda)$ for each $x, y \in C$ and $\lambda \in[0,1]$ (see [20]).

\section{Main results}

In this section the existence of a coincidence point for $(\alpha, \beta, \gamma, \eta)$-generalized contraction pairs is established. We also give some common fixed point theorems for weakly commuting pairs as well as Banach operator pairs in a convex metric space which are $(\alpha, \beta, \gamma, \eta)$ generalized contraction pairs. In what follows, $X$ denotes a convex metric space with the metric $d$ and the convex structure $W$.

The following lemma is a key result to prove Theorem 2.2.

Lemma 2.1 [21] Let $(X, d)$ be a convex metric space. Then

$$
d\left(x, W\left(x, y, \frac{1}{2}\right)\right)=d\left(y, W\left(x, y, \frac{1}{2}\right)\right)=\frac{1}{2} d(x, y)
$$

for all $x, y \in X$.

The following theorem is our main result and plays an important role to prove the next results. 
Theorem 2.2 Let $C$ be a nonempty subset of a convex metric space $X$, and let $S$ and $T$ be two self-mappings of $C$ such that $S(C)$ is convex complete and $T(C) \subseteq S(C)$. If $(S, T)$ is an $(\alpha, \beta, \gamma, \eta)$-generalized contraction pair, then $S$ and $T$ have a coincidence point.

Proof Since $(S, T)$ is an $(\alpha, \beta, \gamma, \eta)$-generalized contraction pair, we have

$$
\alpha d(T x, T y)+\beta(d(S x, T x)+d(S y, T y))+\gamma(d(S x, T y)+d(S y, T x)) \leq \eta d(S x, S y)
$$

for all $x, y \in C$, where $\alpha, \beta, \gamma, \eta \in \mathbb{R}$,

$$
2 \beta+\gamma-|\gamma|-\alpha \leq \eta<\alpha+4 \beta+3 \gamma-|\gamma|, \quad \beta+\gamma \leq 0 .
$$

Let $x_{0}$ be an arbitrary point of $C$. Since $S(C)$ is convex, we can inductively define a sequence $\left\{S x_{n}\right\}_{n=1}^{\infty}$ in $S(C)$ by

$$
S x_{n}=W\left(S x_{n-1}, T x_{n-1}, \frac{1}{2}\right), \quad n \in \mathbb{N} .
$$

By Lemma 2.1 and (2.2), we have

$$
\begin{aligned}
& d\left(S x_{n}, T x_{n}\right)=2 d\left(S x_{n}, S x_{n+1}\right), \\
& d\left(S x_{n}, T x_{n-1}\right)=d\left(S x_{n-1}, S x_{n}\right)
\end{aligned}
$$

for all $n \in \mathbb{N}$. Now by substituting $x$ with $x_{n-1}$, and $y$ with $x_{n}$ in (2.1), we get

$$
\begin{aligned}
& \alpha d\left(T x_{n-1}, T x_{n}\right)+\beta\left(d\left(S x_{n-1}, T x_{n-1}\right)+d\left(S x_{n}, T x_{n}\right)\right) \\
& \quad+\gamma\left(d\left(S x_{n-1}, T x_{n}\right)+d\left(S x_{n}, T x_{n-1}\right)\right) \\
& \leq \eta d\left(S x_{n-1}, S x_{n}\right)
\end{aligned}
$$

for all $n \in \mathbb{N}$. The assumptions imply that $\alpha$ is positive; hence, by the triangle inequality, (2.3), (2.4), and (2.5), we have

$$
\begin{aligned}
\alpha\left(2 d\left(S x_{n}, S x_{n+1}\right)-d\left(S x_{n-1}, S x_{n}\right)\right) & =\alpha\left(d\left(S x_{n}, T x_{n}\right)-d\left(S x_{n}, T x_{n-1}\right)\right) \\
& \leq \alpha d\left(T x_{n-1}, T x_{n}\right)
\end{aligned}
$$

for all $n \in \mathbb{N}$. From (2.3), (2.4), (2.5), and (2.6), we obtain

$$
\begin{aligned}
& \alpha\left(2 d\left(S x_{n}, S x_{n+1}\right)-d\left(S x_{n-1}, S x_{n}\right)\right)+2 \beta\left(d\left(S x_{n-1}, S x_{n}\right)+d\left(S x_{n}, S x_{n+1}\right)\right) \\
& \quad+\gamma\left(d\left(S x_{n-1}, T x_{n}\right)+d\left(S x_{n-1}, S x_{n}\right)\right) \\
& \leq \eta d\left(S x_{n-1}, S x_{n}\right)
\end{aligned}
$$

for all $n \in \mathbb{N}$.

We now claim that the following inequality holds:

$$
2(\alpha+\beta+\gamma) d\left(S x_{n}, S x_{n-1}\right) \leq(\eta+\alpha-2 \beta+|\gamma|-\gamma) d\left(S x_{n-1}, S x_{n}\right)
$$

for all $n \in \mathbb{N}$. 
To see this, we consider the two following cases for $\gamma$.

Case 1. $\gamma \geq 0$ :

By the triangle inequality and (2.3), we have

$$
2 \gamma d\left(S x_{n}, S x_{n+1}\right)=\gamma d\left(S x_{n}, T x_{n}\right) \leq \gamma\left(d\left(S x_{n-1}, T x_{n}\right)+d\left(S x_{n-1}, S x_{n}\right)\right)
$$

for all $n \in \mathbb{N}$.

The inequalities (2.7) and (2.9) imply

$$
2(\alpha+\beta+\gamma) d\left(S x_{n}, S x_{n+1}\right) \leq(\eta+\alpha-2 \beta) d\left(S x_{n-1}, S x_{n}\right)
$$

for all $n \in \mathbb{N}$.

Case 2. $\gamma<0$ :

From the triangle inequality, (2.3) and (2.4), we conclude

$$
\begin{aligned}
\gamma\left(d\left(S x_{n-1}, S x_{n}\right)+2 d\left(S x_{n}, S x_{n+1}\right)\right) & =\gamma\left(d\left(S x_{n-1}, S x_{n}\right)+d\left(S x_{n}, T x_{n}\right)\right) \\
& \leq \gamma d\left(S x_{n-1}, T x_{n}\right)
\end{aligned}
$$

for all $n \in \mathbb{N}$. By (2.7) and (2.11), we obtain

$$
2(\alpha+\beta+\gamma) d\left(S x_{n}, S x_{n+1}\right) \leq(\eta+\alpha-2 \beta-2 \gamma) d\left(S x_{n-1}, S x_{n}\right)
$$

for all $n \in \mathbb{N}$. Now inequality (2.8) follows from (2.10) and (2.12).

The assumptions imply that $0 \leq \frac{\eta+\alpha-2 \beta+|\gamma|-\gamma}{2(\alpha+\beta+\gamma)}<1$. Hence, the sequence $\left\{S x_{n}\right\}_{n=1}^{\infty}$ is contractive. So it is a Cauchy sequence in $S(C)$. Since $S(C)$ is complete, there exists $p \in C$ such that $\lim _{n \rightarrow \infty} S x_{n}=S p$. The triangle inequality and (2.3) imply that

$$
d\left(T x_{n}, S p\right) \leq 2 d\left(S x_{n}, S x_{n+1}\right)+d\left(S x_{n}, S p\right)
$$

for all $n \in \mathbb{N}$. It follows $T x_{n} \rightarrow S p$ as $n \rightarrow \infty$. By (2.1), we have

$$
\begin{aligned}
& \alpha d\left(T x_{n}, T p\right)+\beta\left(d\left(S x_{n}, T x_{n}\right)+d(S p, T p)\right) \\
& \quad+\gamma\left(d\left(S x_{n}, T p\right)+d\left(S p, T x_{n}\right)\right) \leq \eta d\left(S x_{n}, S p\right)
\end{aligned}
$$

for all $n \in \mathbb{N}$. In the above inequality letting $n \rightarrow \infty$, we obtain

$$
(\alpha+\beta+\gamma) d(S p, T p) \leq 0 .
$$

Therefore, $(\alpha+\beta+\gamma) d(S p, T p)=0$. Since $\alpha+\beta+\gamma$ is positive, $S p=T p$. So $p \in C(S, T)$, and this completes the proof.

Corollary 2.3 Let $C$ be a nonempty complete convex subset of a convex metric space $X$, and let $T$ be a self-mapping of $C$. If $T$ is an $(\alpha, \beta, \gamma, \eta)$-generalized contraction, then $T$ has a fixed point. Moreover, $T$ has a unique fixed point provided that one of the three conditions: $\beta \leq 0, \gamma>0$ or $\eta<\alpha+2 \gamma$ holds. 
Proof Our assumptions imply that $(I, T)$ is an $(\alpha, \beta, \gamma, \eta)$-generalized contraction pair. By the preceding theorem, $C(I, T)=F(T)$ is a nonempty set. Let $p \in F(T)$.

We now show that $p$ is unique. Let $q \in F(T)$. Since $T$ is an $(\alpha, \beta, \gamma, \eta)$-generalized contraction, we have

$$
\alpha d(T x, T y)+\beta(d(x, T x)+d(y, T y))+\gamma(d(x, T y)+d(y, T x)) \leq \eta d(x, y)
$$

for all $x, y \in C$, where $\alpha, \beta, \gamma, \eta \in \mathbb{R}$ with $2 \beta+\gamma-|\gamma|-\alpha \leq \eta<\alpha+4 \beta+3 \gamma-|\gamma|$ and $\beta+\gamma \leq 0$.

It is easy to see that if $\beta \leq 0$, or $\gamma<0$, then $\alpha+2 \gamma<\eta$. Therefore, we assume that $\alpha+2 \gamma<\eta$. Since $p, q \in F(T)$, from (2.14), we get

$$
(\alpha+2 \gamma) d(p, q) \leq \eta d(p, q)
$$

Since $\alpha+2 \gamma<\eta$, the above inequality implies that $d(p, q)=0$. Hence, $p=q$, and the proof is complete.

The following example shows that there exists an $(\alpha, \beta, \gamma, \eta)$-generalized contraction pair such as $(S, T)$ such that $(T, S)$ is not an $(\alpha, \beta, \gamma, \eta)$-generalized contraction pair.

Example 2.4 Let $X=\mathbb{R}$ with the usual metric. Suppose that $x_{0}$ is an arbitrary point of $X$, and $\alpha, \gamma, \eta \in \mathbb{R}$ with $\alpha+3 \gamma \leq 2 \eta$, and $\gamma<0$. Then the following are true:

(i) It is easy to see that the inequality

$$
\alpha|x-y|+\gamma\left(\left|2 x-y-x_{0}\right|+\left|2 y-x-x_{0}\right|\right) \leq 2 \eta|x-y|
$$

holds for all $x, y \in X$.

(ii) Let $C=[-4, \infty)$, and define the self-mappings $S$ and $T$ of $C$ by

$$
S x=x+2, \quad T x=\frac{x+1}{2} \quad \text { for all } x \in C .
$$

Set $x_{0}=3$, in (2.15). Consequently, we have

$$
\alpha d(T x, T y)+\gamma(d(S x, T y)+d(S y, T x)) \leq \eta d(S x, S y)
$$

for all $x, y \in C$. Therefore, $(S, T)$ is an $(\alpha, 0, \gamma, \eta)$-generalized contraction pair, $T(C) \subseteq S(C), S(C)$ is a complete convex subset of $X$, and -3 is a coincidence point of $S$ and $T$. Moreover, $(S, T)$ is $(7,0,-1,2)$-generalized contraction pair, but $(T, S)$ is not $(7,0,-1,2)$-generalized contraction pair.

(iii) Let $C=[0, \infty)$. Define the self-mapping $T$ of $C$ by $T x=\frac{x+3}{4}, x \in C$, then $F(T)=\{1\}$. Suppose $2 \beta-\alpha \leq \eta<\alpha+4 \beta+2 \gamma, \beta<0, \gamma<0$ and $\alpha<4 \eta$. Then $T$ is an $(\alpha, \beta, \gamma, \eta)$-generalized contraction pair, and 1 is a unique fixed point of $T$.

Theorem 2.5 Let $C$ be a nonempty subset of a convex metric space $X$. Let $S$ and $T$ be two self-mappings of $C$ such that $S(C)$ is a complete convex subset of $X$, and $T(C) \subseteq S(C)$. If $(S, T)$ is a weakly compatible pair and an $(\alpha, \beta, \gamma, \eta)$-generalized contraction pair, then $S$ and $T$ have a unique common fixed point provided that one of the three conditions: $\beta \leq 0$, $\gamma>0$ or $\eta<\alpha+2 \gamma$ holds. 
Proof It is sufficient we assume that $\eta<\alpha+2 \gamma$. Because one can show that if $\beta \leq 0$ or $\gamma>0$, then $\eta<\alpha+2 \gamma$. By Theorem 2.2, $C(S, T)$ is nonempty. Let $v \in C(S, T)$; hence, $S v=$ $T v=w$. Since $(S, T)$ is weakly compatible, $S w=T w$. According to inequality (1.1), we have

$$
\alpha d(T v, T w)+\beta(d(S v, T v)+d(S w, T w))+\gamma(d(S v, T w)+d(S w, T v)) \leq \eta d(S v, S w) .
$$

It follows that $(\alpha+2 \gamma-\eta) d(w, T w) \leq 0$. This implies that $d(w, T w)=0$ because $\alpha+2 \gamma-\eta$ is positive. Therefore, $T w=w$. Hence, $w$ is a common fixed point of $S$ and $T$.

We now show that $w$ is unique. Suppose $p$ is another common fixed point of $S$ and $T$. According to inequality (1.1), we have

$$
\alpha d(T p, T w)+\beta(d(S p, T p)+d(S w, T w))+\gamma(d(S p, T w)+d(S w, T p)) \leq \eta d(S p, S w) .
$$

It follows that $(\alpha+2 \gamma-\eta) d(p, w) \leq 0$. Since $\alpha+2 \gamma-\eta$ is positive, $d(p, w)=0$. Therefore, $p=w$. Hence, $S$ and $T$ have a unique common fixed point.

Theorem 2.6 Let $C$ be a nonempty complete subset of a convex metric space X. Let $S$ and $T$ be two self-mappings of $C$ such that $F(S)$ is a nonempty closed convex subset of $C$. If $(T, S)$ is a Banach operator pair, and $(S, T)$ is an $(\alpha, \beta, \gamma, \eta)$-generalized contraction pair, then $S$ and $T$ have a common fixed point. Moreover, $S$ and $T$ have a unique common fixed point provided that one of the three conditions: $\beta \leq 0, \gamma>0$ or $\eta<\alpha+2 \gamma$ holds.

Proof Since $(S, T)$ is an $(\alpha, \beta, \gamma, \eta)$-generalized contraction pair, we have

$$
\alpha d(T x, T y)+\beta(d(S x, T x)+d(S y, T y))+\gamma(d(S x, T y)+d(S y, T x)) \leq \eta d(S x, S y)
$$

for all $x, y \in C$, where $\alpha, \beta, \gamma, \eta \in \mathbb{R}$ with $2 \beta+\gamma-|\gamma|-\alpha \leq \eta<\alpha+4 \beta+3 \gamma-|\gamma|$, and $\beta+\gamma \leq 0$.

Since $(T, S)$ is a Banach operator pair, $T$ is a self-mapping of $F(S)$. By the above inequality, we get

$$
\alpha d(T x, T y)+\beta(d(x, T x)+d(y, T y))+\gamma(d(x, T y)+d(y, T x)) \leq \eta d(x, y)
$$

for all $x, y \in F(S)$. Now by Corollary 2.3 , the proof is complete.

Corollary 2.7 Let C be a nonempty complete subset of a convex metric space X. Let $S$ and $T$ be two self-mappings of $C$ such that $S$ is an affine map, and $F(S)$ is a nonempty closed subset of C. If $(T, S)$ is a Banach operator pair, and $(S, T)$ is an $(\alpha, \beta, \gamma, \eta)$-generalized contraction pair, then $S$ and $T$ have a common fixed point. Moreover, $S$ and $T$ have a unique common fixed point provided that one of the three conditions: $\beta \leq 0, \gamma>0$ or $\eta<\alpha+2 \gamma$ holds. 


\section{References}

1. Takahashi, T: A convexity in metric spaces and nonexpansive mapping I. Kodai Math. Semin. Rep. 22, 142-149 (1970)

2. Guay, MD, Singh, KL, Whitfield, JHM: Fixed point theorems for nonexpansive mappings in convex metric spaces. In: Proceedings of Conference on Nonlinear Analysis. Lecture Notes in Pure and Applied Mathematics, vol. 80, pp. 179-189. Dekker, New York (1982)

3. Beg, l, Azam, A: Fixed point on star-shaped subsets of convex metric spaces. Indian J. Pure Appl. Math. 18, $594-596$ (1987)

4. Beg, I, Azam, A, Ali, F, Minhas, T: Some fixed point theorems in convex metric spaces. Rend. Circ. Mat. Palermo XL, 307-315 (1991)

5. Shimizu, T, Takahashi, W: Fixed point theorems in certain convex metric spaces. Math. Jpn. 37, 855-859 (1992)

6. Ciric, L: On some discontinuous fixed point theorems in convex metric spaces. Czechoslov. Math. J. 43(188), $319-326$ (1993)

7. Beg, I: Structure of the set of fixed points of nonexpansive mappings on convex metric spaces. Ann. Univ. Mariae Curie-Skłodowska, Sect. A LII, 7-14 (1998)

8. Beg, I: Inequalities in metric spaces with applications. Topol. Methods Nonlinear Anal. 17, 183-190 (2001)

9. Beg, I, Abbas, M: Fixed points and best approximation in Menger convex metric spaces. Arch. Math. 41, 389-397 (2005)

10. Pant, RP: Common fixed points of noncommuting mappings. J. Math. Anal. Appl. 188, 436-440 (1994)

11. Sessa, S: On a weak commutativity condition of mappings in fixed point considerations. Publ. Inst. Math. 32, 149-153 (1982)

12. Jungck, G: Compatible mappings and common fixed points. Int. J. Math. Math. Sci. 9, 771-779 (1986)

13. Jungck, G, Rhoades, BE: Fixed point for set valued functions without continuity. Indian J. Pure Appl. Math. 29(3), 227-238 (1998)

14. Chugh, R, Kumar, S: Common fixed points for weakly compatible maps. Proc. Indian Acad. Sci. Math. Sci. 111, 241-247 (2001)

15. Jungck, G: Common fixed points for commuting and compatible maps on compacta. Proc. Am. Math. Soc. 103 978-983 (1988)

16. Jungck, G: Common fixed point theorems for compatible self maps of Hausdorff topological spaces. Fixed Point Theory Appl. 3, 355-363 (2005)

17. Chen, J, Li, Z: Common fixed-points for Banach operator pairs in best approximation. J. Math. Anal. Appl. 336 1466-1475 (2007)

18. Hussain, $\mathrm{N}$ : Common fixed points in best approximation for Banach operator pairs with Ćirić type /-contractions. J. Math. Anal. Appl. 338, 1351-1363 (2008)

19. Agarwal, RP, O’Regan, D, Sahu, DR: Fixed Point Theory for Lipschitzian-Type Mappings with Applications. Springer, Heidelberg (2009)

20. Hussain, N, Abbas, M, Kim, JK: Common fixed point and invariant approximation in Menger convex metric spaces. Bull. Korean Math. Soc. 48, 671-680 (2008)

21. Moosaei, M: Fixed point theorems in convex metric spaces. Fixed Point Theory Appl. 2012, Article ID 164 (2012). doi:10.1186/1687-1812-2012-164

10.1186/1687-1812-2014-98

Cite this article as: Moosaei: Common fixed points for some generalized contraction pairs in convex metric spaces. Fixed Point Theory and Applications 2014, 2014:98

\section{Submit your manuscript to a SpringerOpen ${ }^{\circ}$ journal and benefit from:}

- Convenient online submission

- Rigorous peer review

Immediate publication on acceptance

- Open access: articles freely available online

- High visibility within the field

- Retaining the copyright to your article 